for the treatment of renovascular hypertension in such patients.

Methods A retrospective search was conducted of a local database of all patients with confirmed Alagille syndrome and renovascular hypertension treated with PTA. Data for each patient was then collected from medical and electronic records.

Results Four patients were identified with Alagille syndrome and renovascular hypertension. In total, 8 PTA procedures were carried out including one for management of renal artery aneurysm. There were no intra or peri-operative complications including significant bleeding. In addition, two patients needed unilateral nephrectomies for non-functioning kidneys. $75 \%$ of patients had improvement in blood pressure at last follow up. There was a peri-procedural rise in serum creatinine of $10-73 \%$ in $57 \%$ of PTA procedures, the majority of which normalised at last follow up.

Conclusions In a tertiary paediatric renovascular centre, PTA can be safely performed in patients with Alagille syndrome. Improvement in blood pressure was observed in $75 \%$ of cases. Furthermore, this study confirms that patients with Alagille syndrome and renovascular hypertension might need more than one PTA and these should be done in a specialist centre with experience in this patient cohort.

\section{G338(P) DERMATOLOGICAL MANIFESTATIONS IN CHILDREN WITH CHRONIC KIDNEY DISEASE IN A TERTIARY CARE CENTER: A CASE-CONTROL STUDY}

${ }^{1} \mathrm{~L}$ Dawman, ${ }^{1} \mathrm{P}$ Kambagiri, ${ }^{2} \mathrm{~V}$ Keshavmurthy, ${ }^{1} \mathrm{~K}$ Tiewsoh. ${ }^{1}$ Pediatrics, Post Graduate Institute of Medical Education and Research, Chandigarh, India; ${ }^{2}$ Dermatology, Venerology and Leprology, Post Graduate Institute of Medical Education and Research, Chandigarh, India

\subsection{6/archdischild-2020-rcpch.291}

Aims To determine the prevalence of various dermatological manifestations in children with CKD.

Methods Prospective, case-control study of 200 CKD children and 200 healthy controls (aged between 3 months - 14 years) was conducted from January 2018 to July 2019. Detailed dermatological examination was performed including skin, hair, nails and oral cavity in both cases and controls and the results were compared.

Results The median age of the cases and controls were 66.95 months and 62.27 months respectively. Majority of the children belonged to CKD Stage 1 (40\%) followed by Stage $3(22 \%)$. Skin, hair and nail manifestations were seen in $94.5 \%, 32.5 \%$, and $41.5 \%$ in the CKD group and $78 \%$, $14.5 \%$ and $22 \%$ respectively in controls ( $p$ value $-<0.0001$, $<0.0001,0.684)$. At least one skin manifestation was seen in $94.5 \%$ of cases and $78 \%$ of controls. The most common skin manifestation among cases was skin pallor (75.5\%) followed by xerosis (48.5\%), atopic diathesis (41.5\%) and pruritus (13\%), whereas among controls, atopic diathesis (46\%) followed by pallor (24\%), xerosis (14\%) and naevii $(13.5 \%)$ was commonly seen. The most common hair manifestations in cases was flag hair (19.5\%), followed by traction alopecia $(5.5 \%)$ and hypertrichosis $(5 \%)$ whereas in controls, traction alopecia $(5 \%)$ followed by flag hair $(3 \%)$ and hypertrichosis $(0.5 \%)$ was seen. The most common nail finding in cases was punctate leukonychia (22\%) followed by ragged cuticles $(18.5 \%)$ and riding of nails $(4 \%)$ whereas, in the control group, ragged cuticles (23\%) followed by punctate leukonychia $(22.5 \%)$ was seen. The most common oral cavity finding in cases was dental staining $(6 \%)$ followed by dental caries (5\%) and dental enamel hypoplasia (4\%) whereas, in controls, dental caries (8.5\%) followed by dental enamel hypoplasia (4\%) and dental staining due to iron $(3.5 \%)$ was seen. Xerosis was found to be significantly associated with higher levels of creatinine $(\mathrm{p}-0.044)$ and hypocalcemia $(\mathrm{p}$ 0.032).

Conclusions Skin pallor, xerosis, atopic diathesis, pruritis, flag hair, traction alopecia, hypertrichosis, punctate leukonychia, ragged cuticles, riding of nails, dental staining, dental caries and dental enamel hypoplasia were the most common dermatological manifestations seen in children with various stages of CKD.

\section{G339(P) A CASE SERIES ON THE PRESENTATION AND MANAGEMENT OF PAEDIATRIC RENAL ABSCESSES}

KK Muneer, M Muorah. Paediatric Nephrology, Birmingham Women's and Children's NHS FT, Birmingham, UK

10.1136/archdischild-2020-rcpch.292

Aim Renal abscesses are relatively uncommon in Paediatrics when compared to the adult population. This may reflect the diagnostic difficulties of such a rare differential in children (in the developed world); its variable clinical presentations, reliance on imaging and non-specific clinical signs. This case series aims to understand the clinical presentation of children with renal abscesses, their possible risk factors, subsequent management and overall outcome.

Methods A retrospective chart review of 8 patients (median, $3 \frac{1}{2}$ years) from the last 13 years (2005-18) with a diagnosis of renal abscess in terms of their potential unifying risk factors, clinical presentation, ultrasound findings, biochemical parameters, microbiological results, management options (surgical drainage vs. medical management) and overall time to recovery.

Results In our cohort, the female to male ratio was 1.6:1. Commonest risk factors were vesicoureteric reflux (25\%) and urinary tract infections (25\%). The common presenting symptoms were fever $(87.5 \%)$, flank pain $(50 \%)$ and generalized abdominal pain (12.5\%). Right sided renal abscesses commonly involved the upper pole (75\%) while left sided abscesses were both upper (50\%) and lower poles (50\%). Mean CRP at presentation was 130 (range, 47-283) mg/L with neutrophilic leukocytosis on blood count. Mean creatinine was 49 (range, 20-139) $\mu \mathrm{mol} / \mathrm{L}$; urea 4.2 (range, 1.6-11) $\mathrm{mmol} / \mathrm{L}$. Commonest pus culture isolate included E.coli $(25 \%)$ and S.aureus (25\%). Average time to radiological resolution for those managed surgically was 4.3 months and 2.5 months for those managed medically (prolonged course of intravenous antibiotics).

Conclusion Clinical presentation of renal abscesses in children is often non-specific. Ultrasound can be used as an initial screening tool. Commonest organisms isolated are S.aureus and E.coli. The time to radiological resolution of abscesses is variable and not improved by surgical intervention. Conservative management should be considered over invasive surgical procedures if the latter can be avoided. 